\title{
Requiem for Nigro or is anal squamous carcinoma still a surgical problem: Abdominoperineal excision rather than a defunctioning stoma?
}

\author{
JC Kloppers, ${ }^{1}$ B Robertson, ${ }^{2}$ PA Goldberg, ${ }^{1}$ EDT Coetzee ${ }^{1}$ \\ ${ }^{1}$ Surgical Gastroenterology Clinic, Division of General Surgery \\ ${ }^{2}$ Department of Radiation Oncology \\ University of Cape Town Health Sciences Faculty, Groote Schuur Hospital
}

Corresponding author: Dr Christo Kloppers (christo.kloppers@uct.ac.za)

\begin{abstract}
Background: Combined multimodal treatment (CMT) is the preferred treatment for anal squamous carcinoma with radical surgery reserved for treatment failure. Some patients require a defunctioning stoma prior to CMT. Successful closure of such a stoma is unlikely. Abdominoperineal excision (APE) may be suitable as primary treatment in these patients.

Objectives: To evaluate organ preservation in the treatment of anal squamous cancer and the closure rate of pre-treatment, temporary diverting colostomy, thereby assessing whether APE could be offered as primary treatment in those requiring a pre-treatment colostomy.

Methods: A retrospective review of all patients with anal squamous carcinoma was undertaken. Patients who required defunctioning colostomies prior to CMT were analysed for potential resectability of tumour prior to CMT and rate of permanent stoma.

Results: One hundred and twenty-five patients were included of which 58 were males. The mean age was 56 years. 107 were treated with curative intent. Six received primary APE and 12 salvage APE. Thirty (22 males) required pretreatment diverting colostomies. Three (10\%) stomas were successfully reversed. Forty-eight (38\%) of the 125 completed treatment with a permanent colostomy. Six patients who needed a stoma prior to CMT were deemed resectable.

Conclusion: Organ preservation was not possible in about a third of patients. Defunctioning stomas prior to CMT were likely to be permanent. We propose that APE could be considered as an alternative in selective cases where the tumour is resectable with low morbidity and a stoma is indicated.
\end{abstract}

Key words: Anal squamous carcinoma, defunctioning stoma, Nigro protocol, abdominoperineal excision

\section{Introduction:}

Squamous cell carcinoma (SCC) of the anal canal is rare. In the United States, for the year 2017, 1100 deaths due to anal SCC were expected with 8200 new diagnoses comprising $2.6 \%$ of gastrointestinal cancers. ${ }^{1}$ The condition comprises $4 \%$ of the total number of cancers of the gastrointestinal tract seen at Groote Schuur Hospital in Cape Town, South Africa. ${ }^{2}$ Ninety percent of anal canal carcinomas are of squamous cell origin. The remainder consists of adenocarcinoma, melanoma, and neuroendocrine tumours of the anal canal. ${ }^{3}$

Abdominoperineal excision (APE) was the standard of care for anal canal squamous cell carcinoma before the introduction of radiotherapy with induction chemotherapy. In 1974, Nigro et al. introduced combined modality treatment
(CMT) as primary therapy for SCC with a series of over 100 patients treated by CMT and a complete pathological response rate of $93 \%{ }^{4}$ The benefit of CMT is sphincter preservation with avoidance of a permanent stoma. Currently, CMT is the recommended first line therapy for invasive SCC of the anal canal. Surgical resection is reserved as salvage therapy for patients with persistent or recurrent disease or for the treatment of complications associated with radiation therapy. ${ }^{5}$ The standard of care in North America and Europe for CMT consists of combination 5-fluorouracil and Mitomycin $\mathrm{C}$ infusion chemotherapy with radiotherapy to a total dose of $50.4 \mathrm{~Gy}$ delivered in 28 fractions. ${ }^{6}$ Survival rates of up to $80 \%$ have been reported with CMT, but up to $30 \%$ of non-surgical patients will eventually undergo APE for treatment failure. ${ }^{7}$ 
Some patients require a defunctioning colostomy prior to CMT to allow safe delivery of treatment with the lowest risk of treatment interruption. Studies suggest that only a few of these stomas will be successfully reversed after CMT.,8-10 APE as primary treatment for patients with SCC of the anus who require defunctioning has not been previously evaluated. If used in suitable patients, it could avoid the prolonged treatment and potential complications associated with CMT. We aimed to investigate the stoma closure rate in patients treated for SCC of the anal canal, specifically investigating those who required defunctioning colostomies prior to CMT in our institution and to assess if APE could be feasible in this selected subgroup.

\section{Patients and methods:}

A retrospective review of all patients with histological diagnoses of anal squamous carcinoma treated at a tertiary referral centre, Groote Schuur Hospital, Cape Town, South Africa, between 1995 and 2012 was conducted. The multidisciplinary team consists of specialist colorectal surgeons, oncologists, diagnostic radiologists, social workers and stoma therapists. All patients received clinical local staging with a careful clinical examination by a colorectal surgeon and radiation oncologist, or examination under anaesthesia, when adequate awake examination was not possible. Contrasted computerised tomography (CT) scan of the abdomen including pelvis and chest X-rays were performed to complete the clinical staging. If inguinal lymphadenopathy was found, fine needle aspiration of such a node was performed.

Demographic data including age at presentation, race and gender were recorded. Where available, risk factors for anal SCC, namely smoking and human immune deficiency virus (HIV) status, were documented. Human papilloma virus (HPV) testing was not routinely performed. Although sexual orientation and sexual practice are important considerations in anal cancer, this information was not part of the original data collection, and was therefore not included in the study.

Tumour characteristics were documented and subdivided into well-, moderately- or poorly differentiated squamous carcinoma. Staging was documented in the standard TNM format of the American Joint Committee on Cancer Staging system (AJCC). ${ }^{11}$ The $4^{\text {th }}$ to $7^{\text {th }}$ editions of this staging system were published over the study period and used in our unit. As the staging of anal squamous carcinoma did not change, this did not influence the accuracy of our results. Both anal canal and anal margin cancers were included in the data collection.

The treatment intent was documented as curative or palliative. Patients with anal margin carcinoma with a lesion smaller than $2 \mathrm{~cm}$ had a wide local excision if it was deemed resectable with preservation of the sphincter. The histology, specifically the resection margin, was documented.

Patients who required defunctioning colostomy prior to CMT were analysed for indication of stoma, potential resectability of tumour prior to $\mathrm{CMT}$, response to treatment and stoma closure rate. To evaluate the resectability of patients requiring defunctioning colostomy prior to CMT the operative notes of the examination under anaesthesia and staging CT were studied to gain insight into surgical resectability.

During the period of this study, the radiation technique and fractionation changed. Patients treated prior to 2008 received split course chemoradiation with a dose of 42.00 to $44.20 \mathrm{~Gy}$ in 20 fractions followed six weeks later by a further $15.00 \mathrm{~Gy}$ in six fractions to the primary tumour if there was $>50 \%$ reduction in tumour size on clinical evaluation. The radiation field was planned using bony landmarks and clinical tumour extent and was delivered with anterior and posterior fields using 60-Cobalt. From 2008 onwards patients were treated with continuous chemoradiation. Mostly three dimensional conformal techniques were used and treatment was delivered by a linear accelerator with 6-18 MV photons. The radiation prescription was 36.00 Gy in 18 fractions to the entire pelvis followed by a further $14.00 \mathrm{~Gy}$ in 7 fractions to the primary tumour and all involved nodes. The chemotherapy regimen was mitomycin $\mathrm{C} 12 \mathrm{mg} / \mathrm{m}^{2}$ on day 1 and 5 and fluorouracil (5FU) $1000 \mathrm{mg} / \mathrm{m}^{2}$ as a continuous infusion on days $1-4$, with the first four fractions, and $5 \mathrm{FU} 1000 \mathrm{mg} / \mathrm{m}^{2}$ with the last four fractions of irradiation. ${ }^{2}$

All patients were reviewed 6 weeks after completing treatment. Patients treated with split course chemoradiation with $<50 \%$ response measured clinically on digital examination were considered for APE. ${ }^{2}$ For patients treated with continuous chemoradiation, follow-up was continued as long as the tumour was decreasing in size. However, if disease persisted at five months, an examination under anaesthesia with biopsy was performed. Presence of squamous carcinoma on biopsy at five months, or disease progression at any stage during follow-up was regarded as treatment failure and salvage APE was offered. If there was clinical complete response, follow-up was three monthly for the first two years and six monthly until five years after completing treatment. Where available patient's outcomes were included to five year follow-up.

Side effects of radiotherapy and severe skin toxicity resulting in treatment interruption were documented. The histology of those requiring salvage APE was reviewed to assess residual disease and resection margins.

Descriptive analyses were used to characterise the study population. The study was approved by the Faculty of Health Sciences Human Research Ethics Committee of the University of Cape Town (HREC 429/2012).

\section{Results:}

One hundred and twenty-five patients with a mean age of 56 $(\mathrm{SD}=13)$ years were analysed during the 17 -year period from 1995 to 2012 . Fifty-eight were (46.4\%) male. The HIV status was reported in 88 patients, and $10(11.4 \%)$ were positive and most patients were smokers $(\mathrm{n}=78)$.

The median tumour diameter was $5.5 \mathrm{~cm}$ (range 0-12 cm). Eighty-eight patients $(70 \%)$ had a tumour greater than $5 \mathrm{~cm}$. Nineteen were staged as T4, 69 as T3, 24 as T2 and 13 as T1. Fourteen tumours were well-differentiated squamous cell 


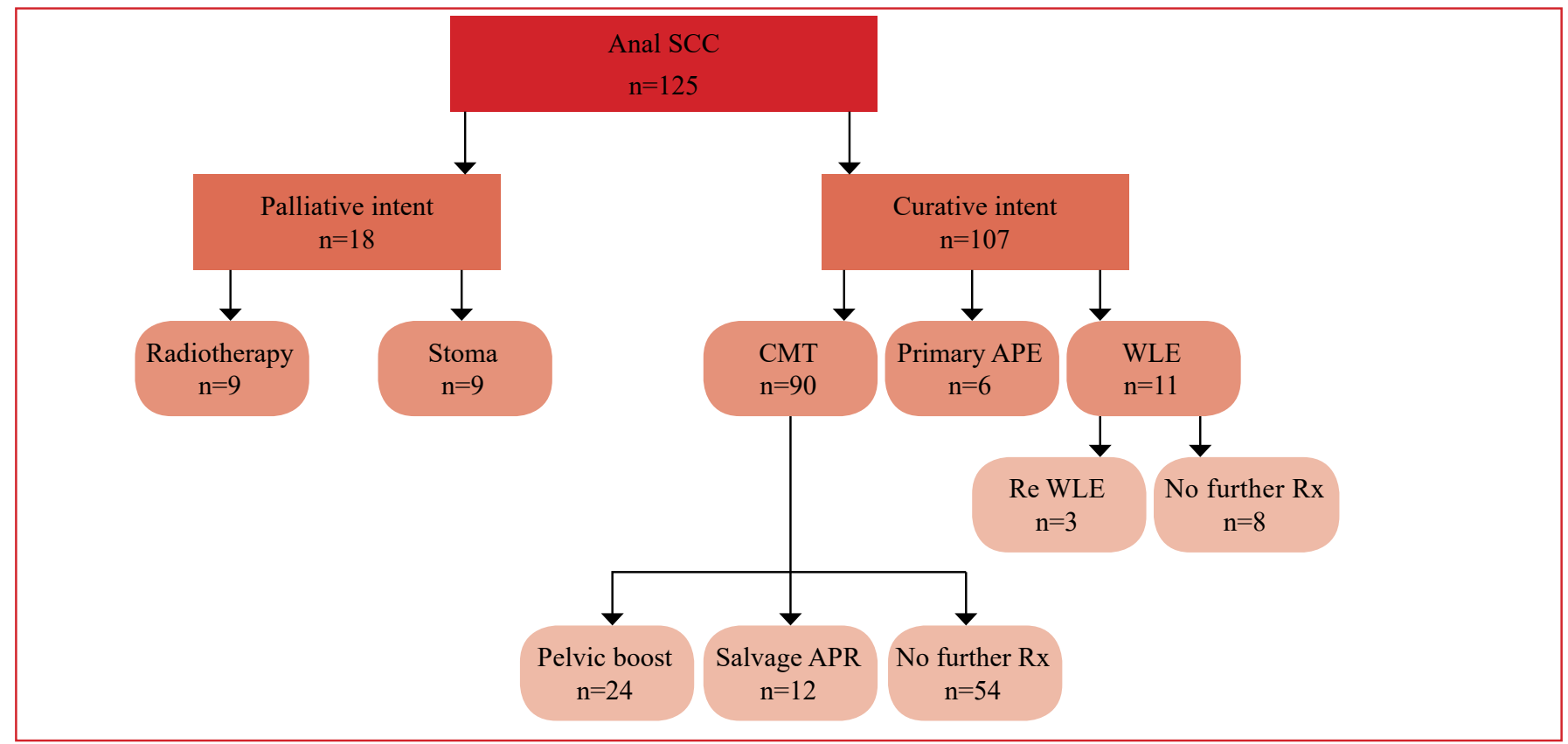

Figure 1: Treatment in anal SCC

carcinoma; 42 were moderately differentiated and 22 poorly differentiated. In 47 patients the degree of differentiation was not reported.

Figure 1 summarises the treatment. One hundred and seven patients received treatment with curative intent. The rest were offered palliation, either with a palliative stoma or radiotherapy. The main indications for palliation were advanced or systemic disease and patients unfit for curative therapy.

Patients with anal margin cancer $(n=11)$ were treated surgically with wide local excision (WLE). Three needed a second WLE after surgical margins were noted to be involved after the first surgery. None of these patients required a stoma.

Six patients were offered primary APE as treatment for their cancer because they were either deemed too frail to undergo CMT $(n=3)$, had previous radiation therapy to the pelvis $(n=2)$ or there was complete destruction of the sphincter complex with incontinence $(\mathrm{n}=1)$. Complete $\mathrm{R} 0$ resection was achieved in all but one.

Ninety patients with anal canal cancer were treated with CMT. Twenty-four patients received a pelvic boost dose after evaluation of response on 6 weeks. In 14 patients radiation was interrupted due to skin-related complications.

Salvage APE was offered to 12 patients of which three were staged T2N0, five staged T3N0, three staged T3N1 and one as $\mathrm{T} 4 \mathrm{~N} 2$ on pre-treatment staging. In nine patients a $\mathrm{R} 0$ resection was achieved, but four developed local recurrence. In three patients a clear surgical margin was not achieved at salvage surgery. The histology of all APEs performed is summarised in Figure 2.

Thirty patients required defunctioning colostomies prior to CMT with the most common indications being obstruction and incontinence (Table 1). Three patients from this subgroup needed salvage APE. Only $4(13 \%)$ of these stomas were eventually reversed, but 1 patient had the stoma restored for incontinence post reversal. Twenty-four $(80 \%)$ of the patients

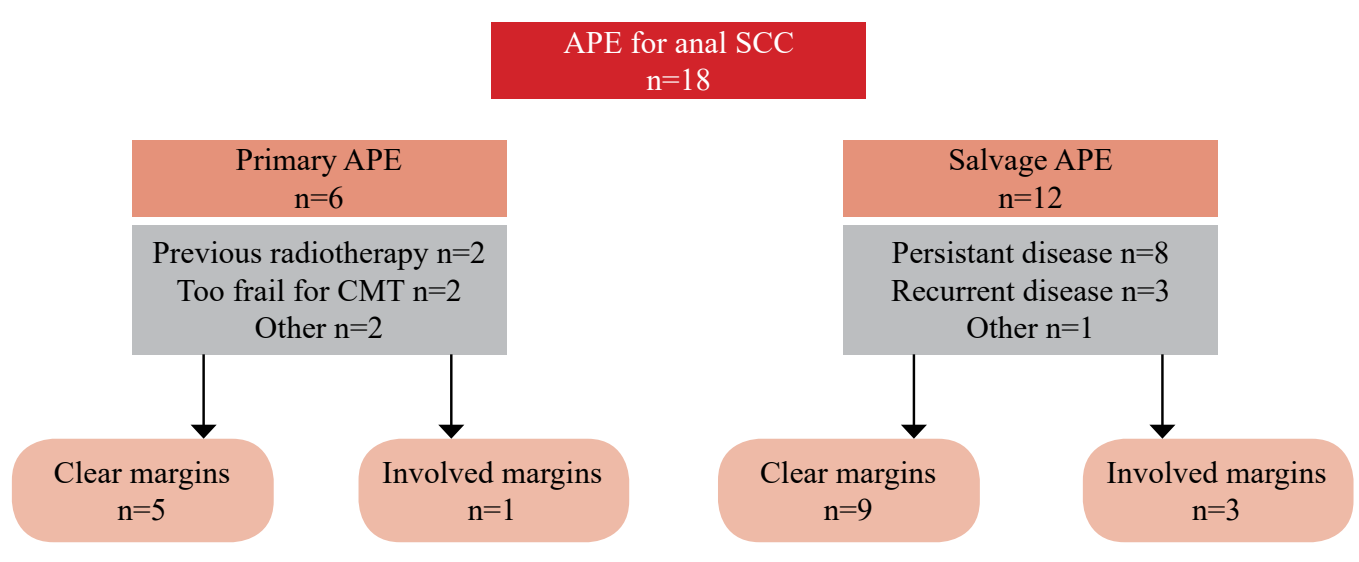

Figure 2: Histology of patients receiving APE for anal SCC 
Table 1: Indications for pre-treatment stomas

\begin{tabular}{lccc} 
Table 1: Indications for pre-treatment stomas & $\begin{array}{c}\text { Cooper et al. 2010 } \\
\text { 35 of } \mathbf{3 4 4} \text { patients }^{\mathbf{8}}\end{array}$ & $\begin{array}{c}\text { Sunesen et al. 2011 } \\
\mathbf{2 0} \text { of 235 patients }\end{array}$ & $\begin{array}{c}\text { Kloppers et al. 2018 } \\
\mathbf{3 0} \text { of 125 patients }\end{array}$ \\
\hline Vaginal fistula & 15 & 6 & $\mathbf{3}$ \\
Obstruction & 1 & 4 & $\mathbf{1 4}$ \\
Incontinence & 10 & 5 & $\mathbf{8}$ \\
Abscess/sepsis & 4 & 4 & $\mathbf{1}$ \\
Pain & 5 & 1 & $\mathbf{4}$
\end{tabular}

requiring a pre-treatment stoma ended up with a permanent colostomy (Figure 3). The reason for non-reversal in 15 of these patients was disease progression. Three were lost to follow-up and it is presumed that they still had their stomas. Three patients died. Inability to maintain continence in preclosure testing $(n=2)$, anal stenosis $(n=1)$ and unknown reasons $(n=4)$ were documented as indications for nonclosure.

Organ preservation is an important aim in the modern treatment of anal squamous cancer. Forty-eight of the 125 patients $(38 \%)$ had a permanent stoma at the end of the treatment (Table 2). Despite radical CMT being standard of care half of patients with anal SCC will still require a surgical procedure as seen in Table 3.

\section{Table 2: Permanent stoma in anal SCC}

\begin{tabular}{lc}
\hline Non-reversed defunctioning stoma & 24 \\
Palliative stoma & 9 \\
Salvage APE & 9 \\
Primary APE & 6 \\
Permanent stoma & 48 from $125(38 \%)$
\end{tabular}

\begin{tabular}{lc} 
Table 3: Surgery for anal SCC \\
\hline Curative intent \\
Primary APE & 6 \\
WLE & 11 \\
Pre-treatment stoma & 30 \\
Salvage APR & 9 \\
Palliative intent & \\
Stoma & 9 \\
Patients needing surgery for anal SCC & 65 from 125(52\%)
\end{tabular}

\section{Discussion:}

SCC of the anus remains rare although the incidence rate of invasive anal carcinoma in the United States increased by approximately 1.9 -fold for men and 1.5-fold for women from 1973-1979 to $1994-2000 .^{12,13}$ It is more common in women and usually occurs in the sixth or seventh decade of life. ${ }^{5}$ The first published series of Africa in 1981 documented 55 cases over 20 years seen at Groote Schuur Hospital, a large urban teaching hospital. ${ }^{14} \mathrm{~A}$ recent review at the same institution reported 31 patients over four years. The median age was

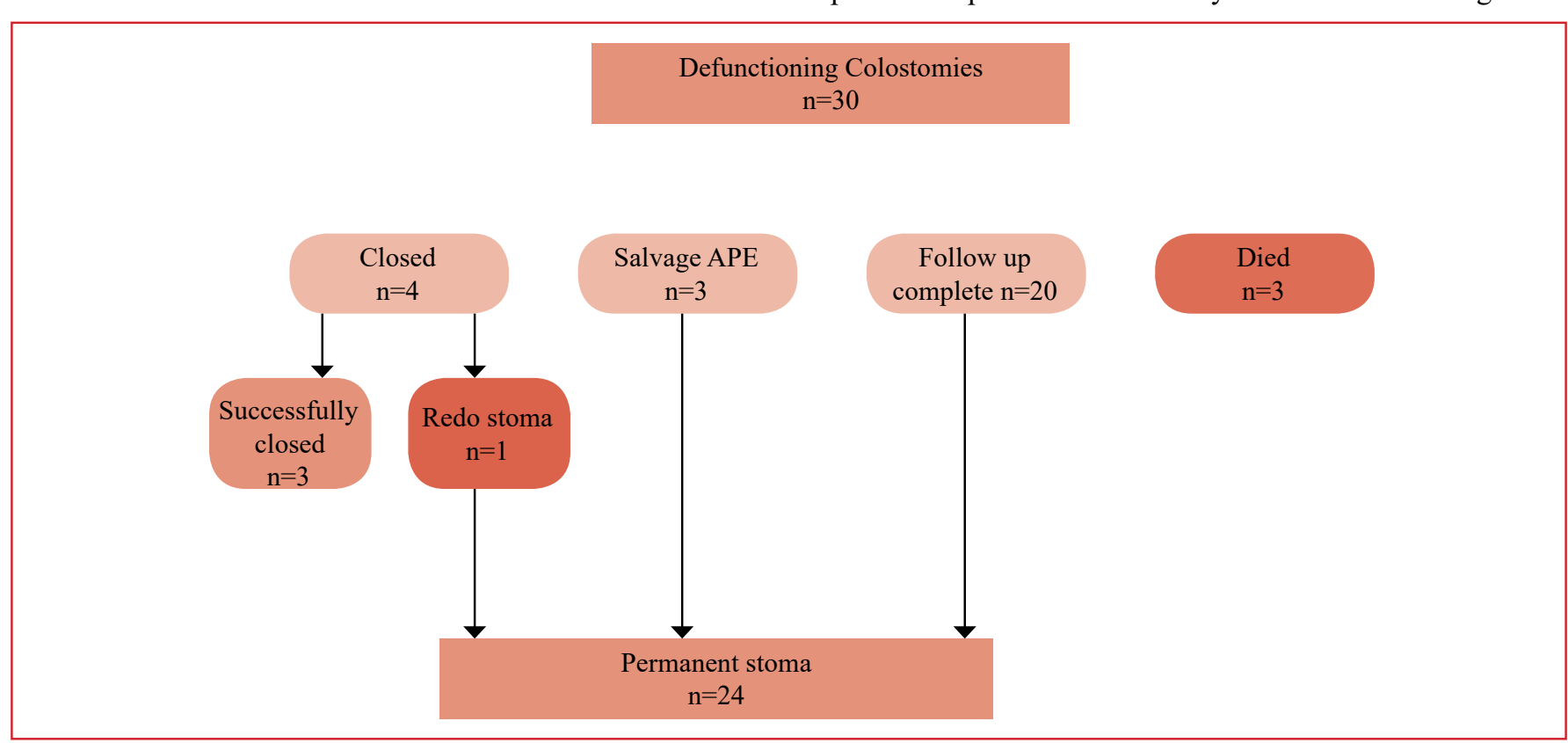

Figure 3: Outcomes of patients requiring pre-treatment diverting colostomy 
56 years (range 18-87). There were 15 females and 16 males. ${ }^{2}$ In this review, anal cancer was calculated to represent $4 \%$ of all gastrointestinal tract cancers which is in contrast to the much lower rates in the developed world. The current series reports on 125 cases over a 17-year period.

CMT is currently the standard of care for patients with anal canal SCC with similar cure rates reported as for APE. CMT has the potential of natural orifice preservation and avoidance of a permanent stoma. This is achievable in the majority of patients with anal canal SCC who are treated with curative intent. $^{12}$

Madden et al. reported a case series including 55 patients from our institution during the APE era, prior to the introduction of CMT as standard of care. They showed that even large tumours of the anal margin could be resected with a fair prognosis. However, the early intra-pelvic spread of carcinoma of the anal canal prevented APE from achieving a high cure rate. ${ }^{14}$ In our series, five of the six patients who received an APE as primary treatment for anal SCC achieved clear surgical margins without significant perioperative morbidity. In the largest series of APE for SCC Lefèvre et al. analysed 105 cases. The mortality rate two months after surgery was $2.1 \%(\mathrm{n}=2)$. Also, 35 patients had at least one complication $(33.3 \%)$, resulting in 21 re-operations $(20 \%){ }^{7}$ Although radical surgery can be performed with acceptable risk, one needs to accommodate for this in clinical decision making.

CMT for anal SCC is not universally well tolerated. Radiotherapy caused skin toxicity in $41 \%$ of our patients, and $15 \%$ of the patients required treatment interruption because of this. Added to this is the systemic side effect profile of chemotherapy, with related morbidity. This could potentially be avoided with a single admission for surgery. Intensitymodulated radiotherapy (IMRT) allows for safe tumour dose escalation while reducing the dose to surrounding normal tissues like skin, small bowel, bladder, femoral heads, external genitalia, and bone marrow. ${ }^{15}$ These newer techniques were not employed during our study period and would potentially lead to less treatment interruptions.

The indications for pre-treatment stomas include the presence or risk of fistula formation, imminent bowel obstruction or faecal incontinence; the latter is usually due to destruction of the anal sphincter by tumour..$^{8,9,16}$ The leading indication for our series is obstruction, which is in contrast with published reports, as seen in Table 1. Several authors have shown that defunctioning colostomy prior to CRT is seldom successfully reversed. Cooper et al. investigated 344 patients with anal SCC. Only seven of 35 patients treated with curative intent, who required a pre-treatment stoma, were successfully reversed. ${ }^{8}$ In the series reported by Sunesen et al., none of 10 patients with pre-treatment stomas were successfully reversed. ${ }^{9}$ Only one of the seven patients with pre-treatment stomas reported by De Bree et al. had his stoma successfully reversed. If a defunctioning stoma is required for SCC of the anal canal, it should be regarded as permanent and patients should be counselled accordingly prior to surgery. In our series only $10 \%$ of pre-treatment stomas were successfully reversed.

In six patients who received a pre-treatment stoma, the primary cancer was deemed resectable before the initiation of the CMT. We propose that an APE could be considered in these individuals as an alternative approach. This could avoid the potential side-effects of CMT. Although CMT will remain the first line treatment for all suitable patients, about half of patients with anal SCC will still need a surgical procedure.

This study has several limitations including the retrospective design and relatively small sample size. We did not include cancer-related outcomes as we specifically investigated stoma closure as primary outcome. As CMT is accepted as the gold standard for the treatment of anal canal squamous cancer, prospective assessment of APE as primary treatment for the cohort requiring pre-treatment colostomy with clinically resectable disease was not performed. For the same reason, the criteria for resectability in anal SCC is not clear as this is not considered at the moment. If prospective evaluation is undertaken, one should consider the impact on quality of life and surgical morbidity of radical surgery comparing to relatively minor surgery with CMT. Both groups will, however, have a permanent stoma.

\section{Conclusion:}

In this era where CMT and organ preservation is the standard of care, this was not possible in about a third of patients treated for anal squamous carcinoma. Defunctioning stoma prior to CMT is likely to be permanent and patients should be counselled to this effect. We propose that for patients requiring a stoma, an APE could be considered as an alternative in selective cases where the tumour is potentially resectable with acceptable morbidity.

\section{Conflicts of interest:}

The authors declare no conflict of interest.

\section{REFERENCES:}

1. Siegel RL, Miller KD, Fedewa SA, et al. Colorectal cancer statistics. CA Cancer J Clin. 2017;67(3):177-93.

2. Robertson B, Shepherd L, Abratt RP, Hunter A, Goldberg P. Treatment of carcinoma of the anal canal at Groote Schuur Hospital. S Afr Med J. 2012;102(6):559-61.

3. Eng C. Carcinoma of the anal canal: Small steps in treatment advances. Clin Adv Hematol Oncol. 2011;9(9):662-9.

4. Nigro ND, Vaitkevicius VK, Considine B Jr. Combined therapy for cancer of the anal canal: A preliminary report. 1974. Dis Colon Rectum. 1993;36(7):709-11.

5. Rousseau DL Jr, Thomas CR Jr, Petrelli NJ, Kahlenberg MS. Squamous cell carcinoma of the anal canal. Surg Oncol. 2005;14(3):121-32.

6. Glynne-Jones R, Northover J, Oliveira J, ESMO Guidelines Working Group. Anal cancer: ESMO clinical recommendations for diagnosis, treatment and follow-up. Ann Oncol. 2009;20 
(Suppl 4):57-60

7. Lefevre $\mathrm{JH}$, Corte $\mathrm{H}$, Tiret E, et al. Abdominoperineal resection for squamous cell anal carcinoma: Survival and risk factors for recurrence. Ann Surg Oncol. 2012;19(13):4186-92.

8. Cooper R, Mason M, Finan P, Byrne P, Sebag-Montefiore D. Defunctioning stomas prior to chemoradiation for anal cancer are usually permanent. Colorectal Dis. 2012;14(1):87-91.

9. Sunesen KG, Norgaard M, Lundby L, et al. Cause-specific colostomy rates after radiotherapy for anal cancer: A danish multicentre cohort study. J Clin Oncol. 2011;29(26):3535-40.

10. de Bree E, van Ruth S, Dewit LG, Zoetmulder FA. High risk of colostomy with primary radiotherapy for anal cancer. Ann Surg Oncol. 2007;14(1):100-8.

11. Edge SB, Compton CC. The American joint committee on cancer: The 7th edition of the AJCC cancer staging manual and the future of TNM. Ann Surg Oncol. 2010;17(6):1471-4.
12. Blumetti J, Bastawrous AL. Epidermoid cancers of the anal canal: Current treatment. Clin Colon Rectal Surg. 2009;22(2):77-83.

13. Johnson LG, Madeleine MM, Newcomer LM, Schwartz SM, Daling JR. Anal cancer incidence and survival: The surveillance, epidemiology, and end results experience, 1973-2000. Cancer. 2004;101(2):281-8.

14. Madden MV, Elliot MS, Botha JB, Louw JH. The management of anal carcinoma. Br J Surg. 1981;68(4):287-9.

15. Shridhar R, Shibata D, Chan E, Thomas CR. Anal cancer: Current standards in care and recent changes in practice. $C A$ Cancer J Clin. 2015;65(2):139-62.

16. Nguyen WD, Mitchell KM, Beck DE. Risk factors associated with requiring a stoma for the management of anal cancer. Dis Colon Rectum. 2004;47(6):843-6. 